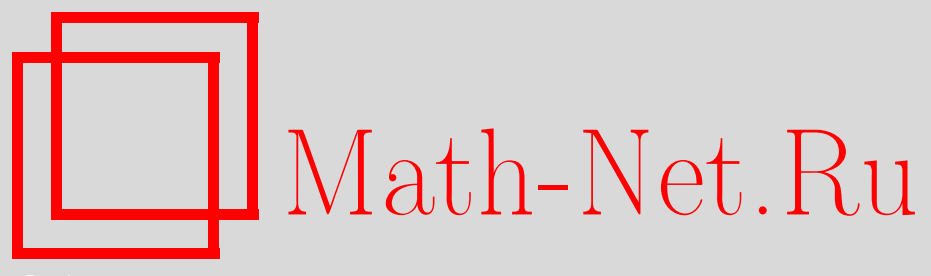

В. Ю. Протасов, Уравнения самоподобия и $p$-радиус операторов, УМH, 2007, том 62, выпуск 6, 185-186

DOI: https://doi.org/10.4213/rm7486

Использование Общероссийского математического портала Math-Net.Ru подразумевает, что вы прочитали и согласны с пользовательским соглашением http://www . mathnet.ru/rus/agreement

Параметры загрузки:

IP : 3.89 .197 .203

26 апреля 2023 г., 15:44:04

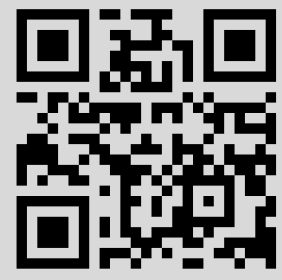




\section{Уравнения самоподобия и $p$-радиус операторов}

\section{В. Ю. Протасов}

Дано конечное семейство линейных операторов $\mathscr{B}=\left\{B_{1}, \ldots, B_{m}\right\}$ в $\mathbb{R}^{d}$, задана некоторая норма $|\cdot|$ в $\mathbb{R}^{d}$ и соответствующая операторная норма $\|B\|=\sup _{|x|=1}|B x|$. Для $k \in \mathbb{N}$ и последовательности $\sigma \in\{1, \ldots, m\}^{k}$ положим $\Pi_{\sigma}=B_{\sigma(1)} \cdots B_{\sigma(k)}$ и обозначим $\mathscr{F}_{k}(p, \mathscr{B})=\left[m^{-k} \sum_{\sigma}\left\|\Pi_{\sigma}\right\|^{p}\right]^{1 / p}$ при $p \in[1,+\infty), \mathscr{F}_{k}(\infty, \mathscr{B})=\max _{\sigma}\left\|\Pi_{\sigma}\right\|$ (усредненная в $L_{p}$ норма произведений длины $k$ операторов семейства $\mathscr{B}$ ).

ОПредЕление 1. $L_{p}$-спектралъным радиусом (далее-p-радиусом) семейства $\mathscr{B}$ называется величина $\rho_{p}=\rho_{p}(\mathscr{B})=\lim _{k \rightarrow \infty}\left[\mathscr{F}_{k}(p, \mathscr{B})\right]^{1 / k}$.

Данный предел всегда существует и не зависит от нормы в $\mathbb{R}^{d}$. При $m=1$ величины $\rho_{p}(\mathscr{B})$ равны обычному спектральному радиусу $\rho\left(B_{1}\right)$. Понятие $p$-радиуса нашло множество применений (см. библиографию в [1]-[6]). Алгоритмы приближенного вычисления $\rho_{p}$ см. в [4]-[6]. Для целых четных $p$ величина $\rho_{p}$ вычисляется явно [4].

Для произвольного $u \in \mathbb{R}^{d}$ положим $\mathscr{F}_{k}(p, u)=\mathscr{F}_{k}(p, \mathscr{B}, u)=\left[m^{-k} \sum_{\sigma}\left|\Pi_{\sigma} u\right|^{p}\right]^{1 / p}$ (с обычным изменением для $p=\infty$ ). Это - усредненная в $L_{p}$ норма образа вектора $u$ под действием всевозможных произведений длины $k$ операторов семейства $\mathscr{B}$. Для $k=1$ полагаем $\mathscr{F}=\mathscr{F}_{1}: \mathscr{F}(|\cdot|, p, \mathscr{B}, u)=\left(m^{-1} \sum_{j=1}^{m}\left|B_{j} u\right|^{p}\right)^{1 / p}, \mathscr{F}(|\cdot|, \infty, \mathscr{B}, u)=$ $\max _{j}\left|B_{j} u\right|$. Далее для краткости пишем $\mathscr{F}(|\cdot|, u)$ вместо $\mathscr{F}(|\cdot|, p, \mathscr{B}, u)$. Норма $|\cdot|$ в $\mathbb{R}^{d}$ называется экстремалъной для $\mathscr{B}$ и для $p$, если существует $\lambda$ такое, что $\mathscr{F}(|\cdot|, u)=\lambda|u|$ при всех $u \in \mathbb{R}^{d}$.

Теорема 1. Неприводимое семейство $\mathscr{B}$ для каждого $p \in[1,+\infty]$ имеет экстремалъную норму. Для любой экстремалъной нормы $\lambda=\rho_{p}(\mathscr{B})$.

Напомним, что семейство неприводимо, если его операторы не имеют общего нетривиального действительного инвариантного подпространства. Экстремальная норма может быть не единственной.

СледСтвие 1. Если $\mathscr{B}$ неприводимо, то $\mathscr{F}_{k}(p, u) \asymp|u|\left(\rho_{p}\right)^{k}, k \in \mathbb{N}$.

Пусть $\mathscr{N}$ - множество всех норм в $\mathbb{R}^{d}$, оно является выпуклым конусом. Имеем отображение конуса $\mathscr{N}$ в себя: $|\cdot| \rightarrow \mathscr{F}(|\cdot|, u)$. По теореме 1 этот нелинейный оператор имеет собственный вектор (возможно, не единственный) с собственным значением $\rho_{p}(\mathscr{B})$. Таким образом, $p$-радиус также выражается через собственное значение, хотя и бесконечномерного нелинейного, оператора. Для приводимых операторов инвариантной нормы может не быть, однако имеет место более слабое утверждение:

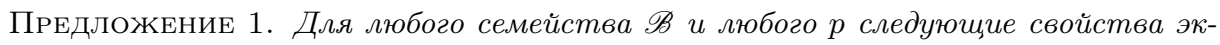
вивалентны:

(a) $\rho_{p}(\mathscr{B})<1$;

(b) существуют норма $|\cdot|$ в $\mathbb{R}^{d} u \gamma<1$ такие, что $\mathscr{F}(|\cdot|, u) \leqslant \gamma|u|, u \in \mathbb{R}^{d}$.

Теорема 1 и предложение 1 (в случае $p=\infty$ они были известны [3]) позволяют получить асимптотику величины $\mathcal{F}_{k}(p, u)$ при $k \rightarrow \infty$. Для любого семейства $\mathscr{B}$ существует базис в $\mathbb{R}^{d}$, в котором матрицы $B_{j}$ имеют блочный нижне-треугольный вид с диагональными блоками $B_{j}^{1}, \ldots, B_{j}^{n}$, причем семейство $\mathscr{B}^{i}=\left\{B_{1}^{i}, \ldots, B_{m}^{i}\right\}$ неприводимо для любого $i$. Тогда $\rho_{p}(\mathscr{B})=\max _{i} \rho_{p}\left(\mathscr{B}^{i}\right)[4]$. Число индексов $i \in\{1, \ldots, n\}$, для которых максимум достигается, назовем валентностью $\mathscr{B}$. Для данного $u \in \mathbb{R}^{d}$ через $V_{u}$ обозначим наименьшее общее инвариантное подпространство операторов $\mathscr{B}$, содержащее $u$.

Работа выполнена при поддержке гранта РФФИ № 05-01-00066 и гранта НШ-5813.2006.1. 
Предложение 2. Даны семейство $\mathscr{B}$ и параметр $p \in[1,+\infty]$. Тогда для любого $u \in \mathbb{R}^{d}$ существуют константы $c_{1}, c_{2}>0$ такие, что $c_{1}|u|\left(\rho_{p}\right)^{k} \leqslant \mathcal{F}_{k}(p, u) \leqslant$ $c_{2}|u| k^{s-1}\left(\rho_{p}\right)^{k}$ при всех $k \geqslant d$, где $\rho_{p}-p$-радиус операторов $\mathscr{B}$, ограниченных на $V_{u}$, $s$ - их валентность на $V_{u}$.

Через $L_{p}[0,1]$ обозначаем пространство вектор-функций из $[0,1]$ в $\mathbb{R}^{d}$ с нормой $\|v\|_{p}=\left(\int_{0}^{1}|v(t)|^{p} d t\right)^{1 / p}$. Пусть в $\mathbb{R}^{d}$ дано семейство аффинных операторов $\tilde{\mathscr{A}}=$ $\left\{\widetilde{A}_{1}, \ldots, \widetilde{A}_{m}\right\}$, которое будем считать неприводимым (не существует общего инвариантного аффинного подпространства, отличного от $\mathbb{R}^{d}$ ). Пусть также дано разбиение отрезка $[0,1]$ точками $0=b_{0}<\cdots<b_{m}=1$; положим $\Delta_{k}=\left[b_{k-1}, b_{k}\right], r_{k}=b_{k}-b_{k-1}$. Аффинная функция $g_{k}(t)=t b_{k}+(1-t) b_{k-1}$ отображает $[0,1]$ в отрезок $\Delta_{k}$. Рассмотрим аффинный оператор самоподобия $\widetilde{\mathbf{A}}$ :

$$
[\widetilde{\mathbf{A}} v](t)=\widetilde{A}_{k} v\left(g_{k}^{-1}(t)\right), \quad t \in \Delta_{k}, \quad k=1, \ldots, m,
$$

определенный в $L_{1}[0,1]$. Уравнение $\widetilde{\mathbf{A}} v=v$ называется уравнением самоподобия. Такие уравнения применяются в эргодической теории, теории всплесков, теории приближений и т. д. (см. библиографию в [1], [2], [5], [7]). Большинство классических фрактальных кривых (Кантора, Кох, де Рама и т. д.) являются решениями уравнений самоподобия. Теорема 1 и предложения 1, 2 позволяют получить точный критерий разрешимости этих уравнений в $L_{p}$ и $C$ и оценить гладкость решений. Для аффинного оператора $\widetilde{A}$ обозначаем через $A$ его линейную часть; для $z=\left(z_{1}, \ldots, z_{m}\right) \in \mathbb{R}_{+}^{m}$ и $\alpha \in \mathbb{R}$ обозначим $z^{\alpha} \mathscr{A}=\left\{z_{1}^{\alpha} A_{1}, \ldots, z_{m}^{\alpha} A_{m}\right\}$. Пусть также $r=\left(r_{1}, \ldots, r_{m}\right)$.

Теорема 2. Уравнение самоподобия имеет решение $v \in L_{1}$ тогда и только тогда, когда $\rho_{1}(m r \mathscr{A})<1$. Это решение единственно.

Если для данного $p \in[1,+\infty]$ выполнено $\rho_{p}\left((m r)^{1 / p} \mathscr{A}\right)<1$, mо $v \in L_{p}$. При $p<\infty$ верно и обратное: из $v \in L_{p}$ следует $\rho_{p}<1$. Из $v \in L_{\infty}$ следует $\rho_{\infty} \leqslant 1$.

Обратимся к непрерывным решениям $v(t)$. Если $\rho_{\infty}(\mathscr{A})<1$, то операторы семейства $\widetilde{\mathscr{A}}$ - сжимающие в некоторой норме (предложение 1$)$. В частности, каждый $A_{j}$ имеет единственную неподвижную точку $v_{i}$. Семейство $\widetilde{\mathscr{A}}$ удовлетворяет перекрестному условию, если $\widetilde{A}_{j} v_{m}=\widetilde{A}_{j+1} v_{1}$ для всех $j=1, \ldots, m-1$.

Теорема 3. Решение $v(t)$ непрерывно тогда и только тогда, когда $\rho_{\infty}(\mathscr{A})<1$ и выполнено перекрестное условие. Его показатель Гёльдера $\alpha_{v}$ является единственным решением уравнения $\rho_{\infty}\left(r^{-\alpha} \mathscr{A}\right)=1$. Для модуля непрерывности в пространстве $C[0,1]$ имеем $C_{1} h^{\alpha} \leqslant \omega(v, h) \leqslant C_{2}|\ln h|^{s-1} h^{\alpha}$ для любого $h \in(0,1 / 2)$, где $\alpha=\alpha_{v}$, $s$ - валентность семейства $r^{-\alpha} \mathscr{A}$ при $p=\infty$.

\section{Список литературы}

[1] D. Collela, C. Heil, SIAM J. Matrix Anal. Appl., 15:2 (1994), 496-518. [2] K.-S. Lau, J. Wang, SIAM. J. Math. Anal., 26:4 (1995), 1018-1046. [3] Н. Е. Барабанов, Автомат. и Телемех., 1988, №5, 17-24. [4] В. Ю. Протасов, Изв. РАН. Сер. матем., 61:5 (1997), 99-136. [5] В. Ю. Протасов, Изв. РАН. Сер. матем., 70:5 (2006), 123-162. [6] V. D. Blondel, Yu. Nesterov, SIAM J. Matrix Anal. Appl., 27:1 (2005), 256-272. [7] M. Solomyak, E. Verbitsky, Bull. London Math. Soc., 27:3 (1995), 242-248.

В. Ю. Протасов (V. Yu. Protasov)

Московский государственный университет им. М. В. Ломоносова

E-mail: v-protassov@yandex.ru
Представлено В. М. Тихомировым Принято редколлегией 27.07.2007 